\title{
ANALYSIS OF EMBEDDED RETAINING WALL USING THE SUBGRADE REACTION METHOD
}

\author{
TOMASZ PASIK, MAREK CHALECKI, EUGENIUSZ KoDA \\ Warsaw University of Life Sciences - SGGW, Faculty of Civil and Environmental Engineering, \\ ul. Nowoursynowska 159, 02-776 Warszawa, Poland, \\ e-mail: tomasz_pasik@sggw.pl,marek_chalecki@sggw.pl,eugeniusz_koda@sggw.pl
}

\begin{abstract}
This paper analyzes the distribution of internal forces and displacements of embedded retaining wall in Quaternary deposits and Tertiary clays. Calculations have been based on the Subgrade Reaction Method (SRM) for two different types of earth pressure behind the wall (active, at-rest) in order to show the differences resulting from adopting the limit values. An algorithm for calculation of "cantilever wall" using the Mathematica program was proposed.
\end{abstract}

Key words: earth pressure, internal forces, displacements, Subgrade Reaction Method, retaining wall

\section{INTRODUCTION}

Proper analysis of retaining wall, used to support deep excavations, is one of the main geotechnical problems to solve during the process of foundation in urbanized areas [11], [12]. The following approaches are most often used to calculate the internal forces and displacements of embedded retaining walls:

- classical methods (CM),

- Subgrade Reaction Method (SRM),

- Finite Element Method (FEM),

all of which are mentioned in the relevant literature [6], [12], [15].

The Subgrade Reaction Method is often used for structural calculation of embedded walls. This is reflected by the availability on the market of numerical programs, such as GEO5 Sheeting Check, DEEPXCAV, GGU-RETAIN, K-REA, D-SHEET PILING.

In order to show computational procedure for beam on elastic foundation, working as an embedded retaining wall, the following operations have been performed:

- elaboration of a model cross-section for the calculation purposes and defining geometrical dimensions of the construction,

- determination of earth pressure on the retaining wall construction,

- analysis of internal forces and displacement for two variants of earth pressure using the Mathe- matica [13]. The results obtained were compared to GEO5 Sheeting Check [4].

The main problem reported by engineers in using this method is to select a correct coefficient of horizontal subgrade reaction $k_{h}$. This, however, is not the only problem, which will be presented in our paper.

\section{EXAMPLE OF CALCULATIONS}

A model cross-section of a deep excavation shoring in the form of diaphragm walls for the project implemented at Tamka street in Warsaw (Fig. 1) has been used as an example for calculating internal forces and displacement. The results of ground investigation and the selected ground parameters needed to carry out the calculations for Warsaw subway and other structures in Warsaw are outlined in papers [1], [5], [7], [14] prepared by the Department of Geotechnical Engineering of Warsaw University of Life Sciences and presented in Fig. 1.

A static diagram shown in Fig. 5, will consist of a beam in the form of a diaphragm wall at the "cantilever phase" loaded by the resultant earth pressure and supported with "Winkler's springs".

The earth pressure load on retaining structure (Fig. 4) has been determined by means of the GEO5 Sheeting Check program so that the values obtained may be compared with the Mathematica analytical calculations. The pressure was determined for active and 


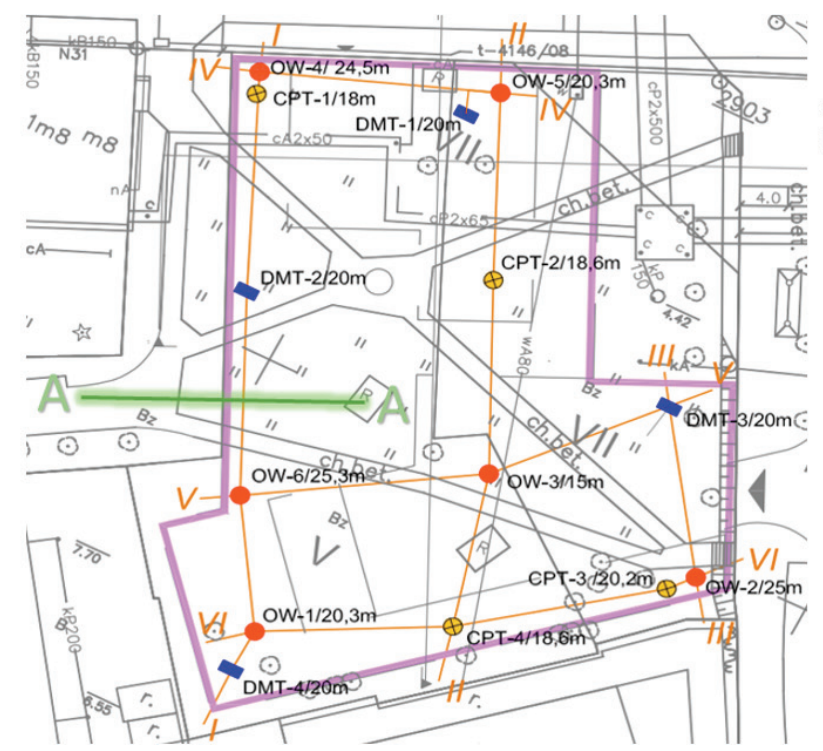

a)



b)

Fig. 1. Cross-section A-A used for calculations of wall construction:

(a) - location of drilling and geotechnical tests [7], (b) - geotechnical layers and material properties

passive state, respectively, based on Coulomb's and Caquot-Kerisel's theory described in [2], [4], [8].

\section{CALCULATION METHOD}

The Subgrade Reaction Method allows the interaction between the embedded retaining structure and the retained earth to be expressed in mathematical terms, while assuming that the structure model has been conceived as a beam supported with a single-parameter Winkler's ground. A scheme of Winkler's model and definition of its basic parameters are shown in Fig. 2. Differential equations of the Winkler's model are described similarly to a regular beam (Euler-Bernoulli equation), except for the continuous load which should be supple- mented with a term resulting from the subgrade reaction $r(x)$ :

Euler-Bernoulli equation

$$
E J \frac{d^{4} w(x)}{d x^{4}}=q_{o}(x)
$$

Winkler equation

$$
\begin{gathered}
E J \frac{d^{4} w(x)}{d x^{4}}=q_{o}(x)-r(x), \\
r(x)=\mathrm{B} \cdot k_{h} \cdot w(x),
\end{gathered}
$$

where $E J$ is the beam stiffness, $\mathrm{B}$ is the beam width, $k_{h}$ is the coefficient of horizontal subgrade reaction, while $w(x)$ and $q_{o}(x)$ are deflection of the beam and load functions, respectively.

In order to determine the internal forces, the following equations must be taken into consideration
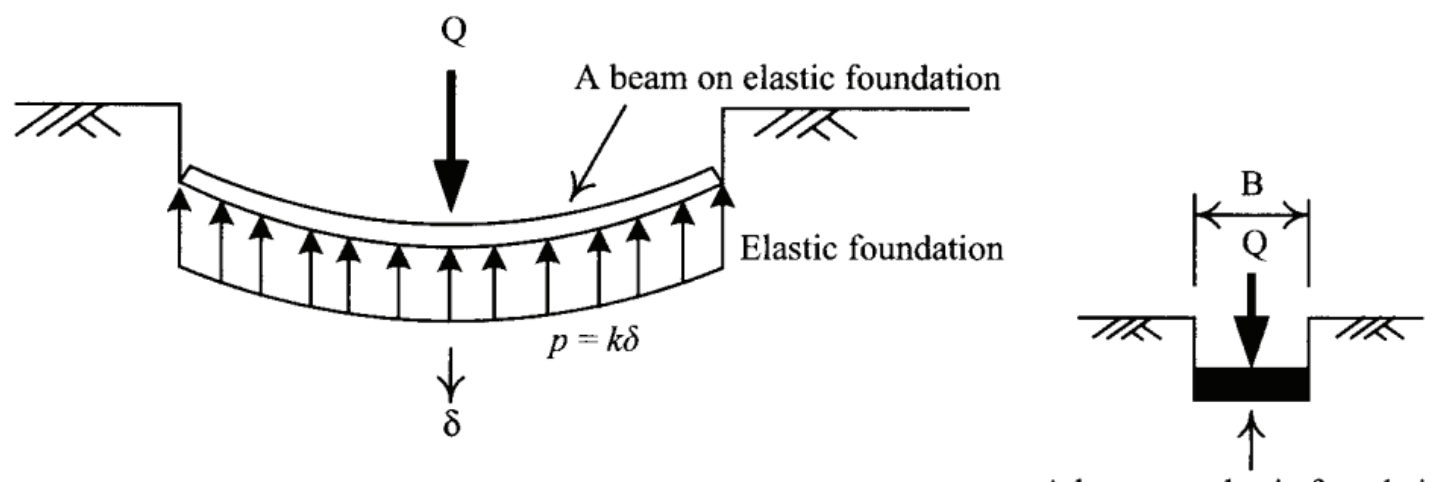

Fig. 2. Winkler's model and basic definition of coefficient $k$, where $p$ stands for stress and $\delta$ stands for displacement [6] 


$$
\begin{aligned}
& E J \frac{d^{2} w(x)}{d x^{2}}=M(x), \\
& E J \frac{d^{3} w(x)}{d x^{3}}=T(x),
\end{aligned}
$$

where $M(x)$ is the bending moment and $T(x)$ is the shearing force.

In order to apply the Winkler model in the context of embedded retaining wall it is crucial to assume that in the range of the state - from active to passive - in accordance with the earth pressure theories, the ground behaves as an elastic material, and after having gone past the critical value it moves into the range of plasticity (Fig. 3a).
The curve illustrating the dependence between earth pressure and displacement is approximated by a straight line, which is described by the horizontal coefficient of subgrade reaction $k_{h}$ (Fig. 3b).

In our case, the value of $k_{h}$ was calculated according to the Chadeisson proposal presented in equation (6) and publications [3], [7], [12]

$$
k_{h}=\left[20 \cdot E J \cdot\left(\frac{K_{p} \cdot \gamma \cdot\left(1-\frac{K_{o}}{K_{p}}\right)^{4}}{0.015}\right)\right]^{\frac{1}{5}}+A_{p} \cdot c^{\prime} \cdot \frac{\operatorname{tgh}\left(\frac{c^{\prime}}{30}\right)}{0.015}
$$

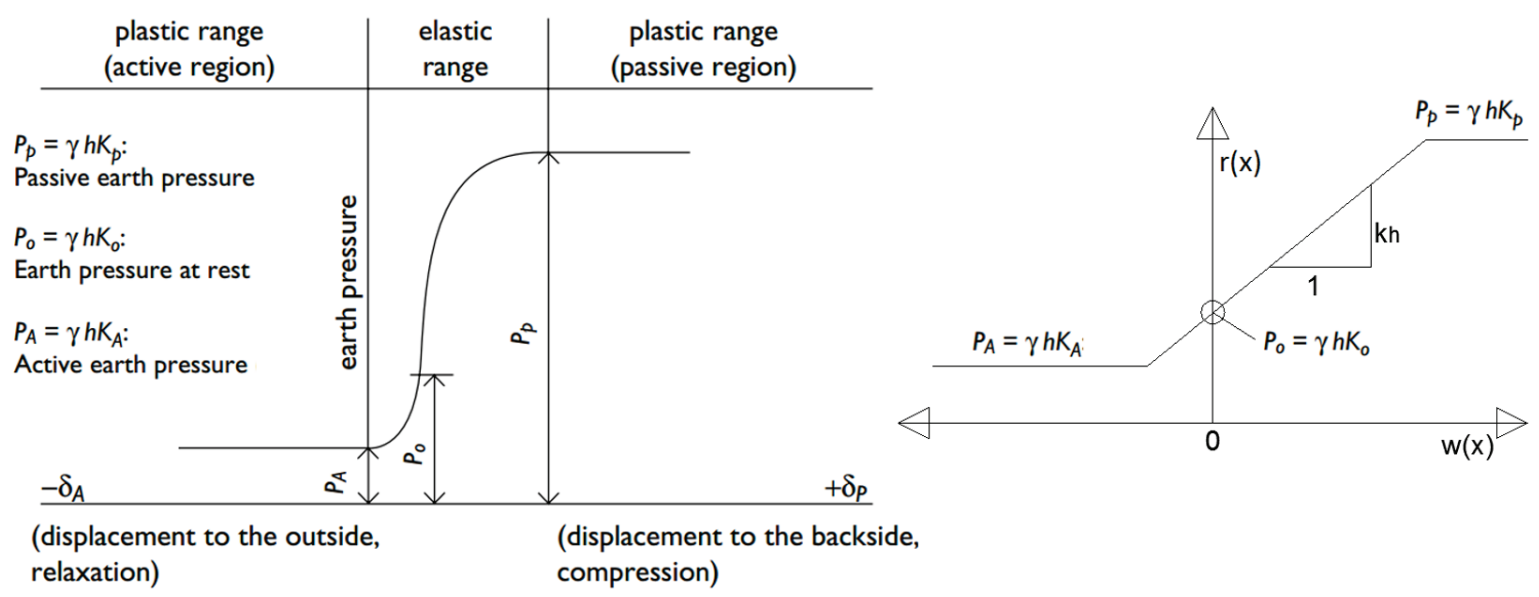

a)

b)

Fig. 3. Variation of the magnitude of lateral earth pressure with wall displacement:

(a) - real dependence [10], (b) - dependence applied to SRM

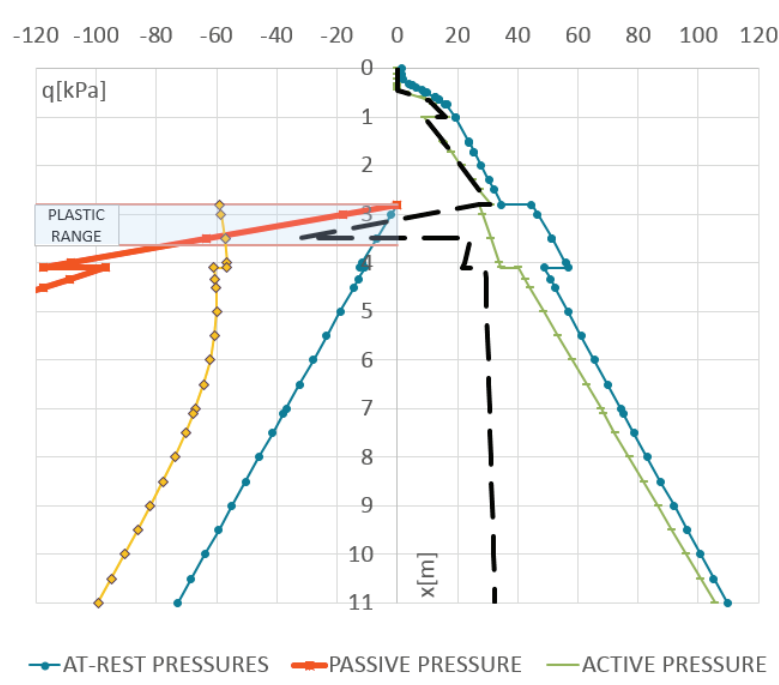

a)

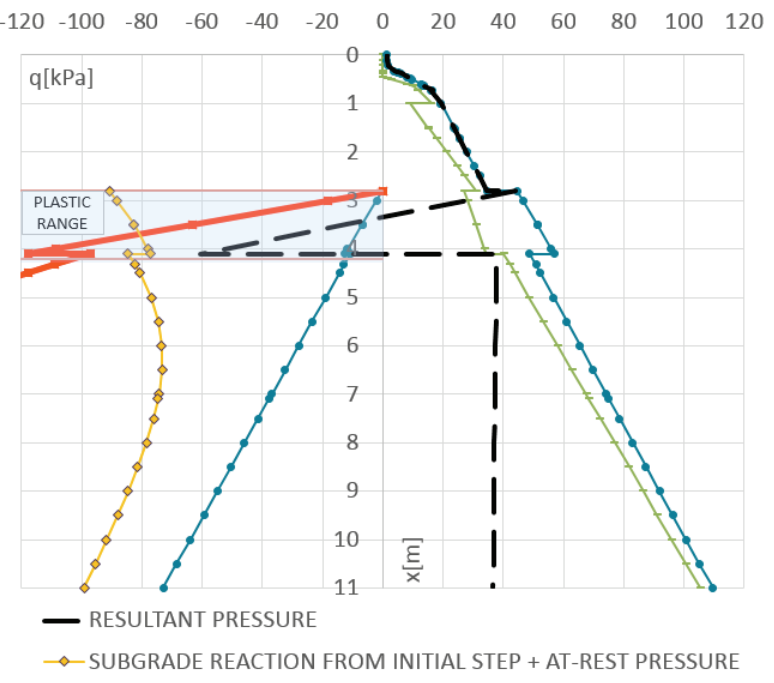

b)

Fig. 4. Pressure exerted on the analyzed retaining wall structure:

(a) - active load behind the structure, (b) - at-rest load behind the structure 


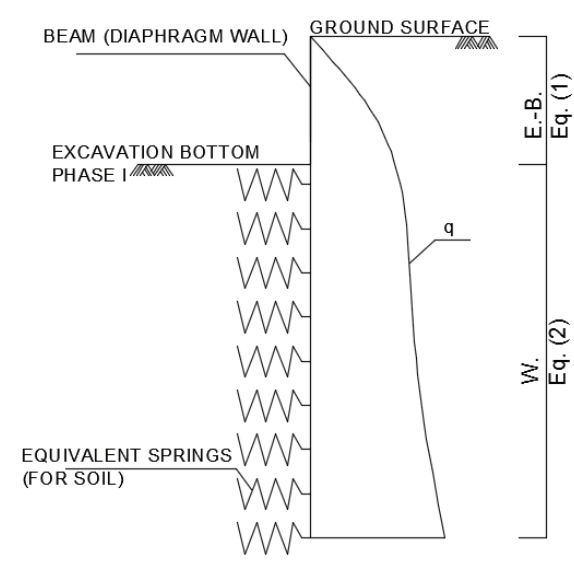

a)



b)

Fig. 5. Static diagram of analyzed retaining wall construction: (a) - initial step, (b) - final step

where

$\gamma-$ specific gravity of soil,

$K_{p}$ - passive pressure coefficient,

$K_{o}$ - pressure coefficient at-rest,

$c^{\prime}$ - effective cohesion,

$A_{p}-$ coefficient allowing for soil cohesion,

$E J$ - beam stiffness.

For the purpose of calculations, it is necessary to determine a load to the beam by summing the figures of earth pressure on both sides of the wall (Fig. 4).

To get results of internal forces and displacements of the analyzed diaphragm wall, it is necessary to determine the scope of the plasticity range of the soil from the side of the excavation. This is done with an initial assumption that in front of the wall the earth pressure behaves elastically from the at-rest state to the passive one, on the whole length of the wall (see Fig. 5, INITIAL STEP).

Next, we move onto the algorithm with the required static scheme and we determine the Winkler reaction $r(x)$ from the dependence of equation 2 or equation 3 , see Fig. 4. The point of intersection of the curves of the Winkler reaction $r(x)$ with the passive earth pressure determines the approximated range of plasticity.

In the next attempt, we repeat the whole algorithm, increasing the zone of plasticity up to the point where, on the boundary between the zone of plasticity and elasticity, the value of the function $E J \frac{d^{4} w(x)}{d x^{4}}$ on the left side will be the same as on the right side (there is "no jump of the function"), see Fig. 10 FINAL, where on the depth of $3.63 \mathrm{~m}$ the graph of the function has one value.

It has been assumed that active earth pressure acts behind the wall. At-rest earth pressure acts in front of the wall, and on the section of the range of plasticity the load passive earth pressure is accepted as the maximum pressure that can be exerted by the soil (see Fig. 4), where the springs of Winkler do not exist. Fig. 5 FINAL STEP.

For comparison, calculations for at-rest earth pressure behind the wall have been made. Figs. 11 -15 .

The calculations using the Mathematica program have been performed based on the following steps:

- The beam was divided into elements;

- The pressure process was approximated using, for example, Legendre polynomials so as to obtain load function $q_{o}(x)$ in the intervals;

- The polynomials were inserted in the Euler-Bernoulli and Winkler's supplemented equations, and solved;

- The constants of integration were determined - for this a system of equations was built based on boundary and continuity conditions;

- The constants of integration were inserted to the general solutions of deflection equations $w(x)$ for each section and then the equations were integrated;

- The internal forces and the diaphragm wall displacements were determined using the equations (2), (4), (5).

\section{CALCULATION RESULTS}

Figures 6-15 show results of calculations for internal forces and displacements. Each plot shows two distributions of a given value. The first is from the authors' own calculations in Mathematica program 










(line), the second - readings from GEO5 Sheeting Check program (dots - point data).

Calculations were made for two types of earth pressure behind the wall, namely: active Figs. 6-10 and for at-rest Figs. 11-15. Thus, the differences, which resulted from applying the extreme values of earth pressure behind the wall, have been shown.

As we can see, the graphs from Mathematica and GEO5 Sheeting Check programs are the same at Figs. 6-10 FINAL STEP, therefore, the calculation approach used is proven correct.

\section{CONCLUSIONS}

The subgrade reaction method is rather more complicated than it seems at first. The designer should be aware of the difficulties encountered at each step of calculations, such as ground investigation, measurement or determination of the soil parameters, $k_{h}$ coefficient(s) $\rightarrow$ calculation of the load based on the selection of relevant pressure theories in relation to the ground type and structure statics $\rightarrow$ assumptions of the subgrade reaction method $\rightarrow$ result $\rightarrow$ checking, verification. Each of the stages mentioned affects the final result.

Measurements of results in real conditions as well as calculations and adjustment of parameters using back analysis are vital for model verification, because only then a given calculation process may be considered correct, depending on compliance with the measurements in real cases.

According to the authors, the inaccuracy of the subgrade reaction method results from:

- The coefficient of horizontal subgrade reaction $k_{h}$ - not only does it result from the difficulty in selecting the proper value assumed throughout the calculations but also from the assumption that it is linear when in reality the dependence between earth pressure and displacement has a non-linear character (Fig. 3).

- The earth pressure assumed for calculations that undergoes redistribution depending on the way the wall is displaced.
In the following sections, the authors make the program code available to allow the reader to check the model assumptions and to follow the calculation procedure. This provides background for discussion, improvement and further exploration, or even development of the SRM.

To simplify the understanding of the code in Table 1 the symbols used in the algorithm have been explained, and in Table 2 the division of beam into elements has been presented.

Table 1. Main designation symbols in algorithm

\begin{tabular}{|l|l|}
\hline Symbols & \multicolumn{1}{|c|}{ Description } \\
\hline d0045 & point data in 0-0.45 interval \\
q0045 & function made from point data in 0-0.45 interval \\
dataQ & earth pressure point data for whole beam \\
q & load function for whole beam \\
w1 & overall displacement function in first element \\
ww1 & special displacement function for first element \\
dataW & displacement point data for whole beam \\
ww & displacement function for whole beam \\
for1 & shearing force function for first element \\
for & shearing force function for whole beam \\
dataF & shearing force point data for whole beam \\
mom1 & bending moment function for first element \\
mom & bending moment function for whole beam \\
dataM & bending moment point data for whole beam \\
pre1 & pressure function for first element \\
pre & pressure function for whole beam \\
dataP & pressure point data for whole beam \\
\hline
\end{tabular}

Table 2. Intervals of beam used in algorithm

\begin{tabular}{|l|c|}
\hline \multicolumn{1}{|c|}{ Intervals } & $\begin{array}{c}\text { Overall displacement functions } \\
\text { for each elements of the beam }\end{array}$ \\
\hline d $0-0.45$ & w1 \\
d $0.45-1$ & w2 \\
d $1-2.3$ & w3 \\
d $2.3-2.8$ & w4 \\
d 2.8-3.5 & w5 \\
d 3.5-4 & w6 \\
d $4-4.1$ & w7 \\
d $4.1-7.1$ & w8 \\
d $7.1-11$ & w9 \\
\hline
\end{tabular}




\section{APPENDIX - MATHEMATICA CODE}

1. Load approximation

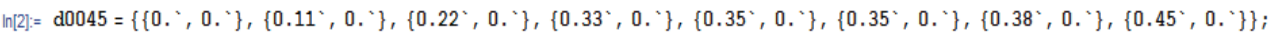
q0045 = LinearModelFit $[$ d0045, $\{\mathbf{x}\}, \mathrm{x}]$;

q0045["BestFit"]

Out $[4]=0$.



$\left.\left\{0.64^{\circ}, 10.23^{\circ}\right\},\left\{0.73^{\circ}, 11.71^{\prime}\right\},\left\{0.73^{\circ}, 11.71^{\circ}\right\},\left\{1 .^{\circ}, 16.06^{\circ}\right\}\right\}$;

q0451 = LinearModelFit $\left[\mathrm{d} 0451,\left\{\mathrm{x}, \mathrm{x}^{2}, \mathrm{x}^{3}\right\}, \mathrm{x}\right]$;

q0451["BestFit"]

Out[l] $=-83.5386+318.005 x-359.418 x^{2}+140.99 x^{3}$

$\ln [8]:=$ Show $[$ Plot $[\mathrm{q} 0451[\mathrm{x}],\{\mathrm{x}, 0.45,1\}$, PlotRange $\rightarrow$ All $]$, ListPlot [d0451, PlotStyle $\rightarrow$ PPointSize $[.02]\}]$, AxesLabel $\rightarrow\{$ "x $[\mathrm{m}] ", " \mathrm{q}[\mathrm{kPa}] "\}]$

$\mathrm{q}[\mathrm{kPa}]$

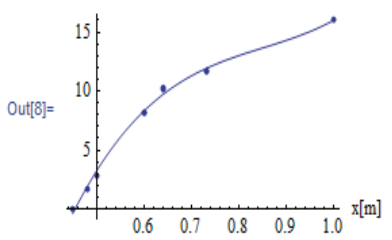

$\ln [9]:=d 123=\left\{\left\{1 .^{`}, 8.97^{`}\right\},\left\{1.5^{`}, 15.08^{`}\right\},\left\{1.52^{`}, 15.34^{`}\right\},\left\{1.72^{`}, 17.81^{`}\right\},\left\{1.72^{`}, 17.82^{`}\right\},\left\{2 .^{\prime}, 21.2^{\prime}\right\},\left\{2.3^{\prime}, 24.86\right\}\right\} ;$ q123 = LinearModelFit $[\mathrm{d} 123,\{\mathrm{x}\}, \mathrm{x}]$;

q123["BestFit"]

Out[11] $=-3.24395+12.2268 \mathrm{x}$

$\ln [12]:=\operatorname{Show}[\mathrm{Plot}[\mathrm{q} 123[\mathrm{x}],\{\mathrm{x}, 1,2.3\}, \mathrm{PlotRange} \rightarrow \mathrm{All}], \operatorname{ListPlot}[\mathrm{d} 123, \mathrm{PlotStyle} \rightarrow\{$ PointSize $[.02]\}]$,

AxesLabel $\rightarrow\{\mathrm{x}[\mathrm{m}] "$, "q[ $[\mathrm{kPa}] "\}]$

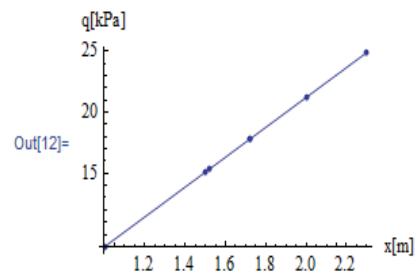

$\ln [13\}]=d 2328=\left\{\left\{2.3^{`}, 24.86\right\},\left\{2.5^{`}, 27.3^{`}\right\},\left\{2.8^{`}, 30.97^{`}\right\}\right\}$

q2328 = LinearModelFit $[\mathrm{d} 2328,\{\mathrm{x}\}, \mathrm{x}]$;

q2328 ["BestFit"]

Jut [15] $=-3.25+12.2211 x$

$\ln [16]=\operatorname{Show}[\mathrm{Plot}[\mathrm{q} 2328[\mathrm{x}],\{\mathrm{x}, 2.3,2.8\}$, PlotRange $\rightarrow$ All], ListPlot [d2328, PlotStyle $\rightarrow\{$ PointSize $[.02]\}]$, AxesLabel $\rightarrow\{" \mathrm{x}[\mathrm{m}] \mathrm{n}, \mathrm{q}[\mathrm{kPa}] \mathrm{n}\}]$

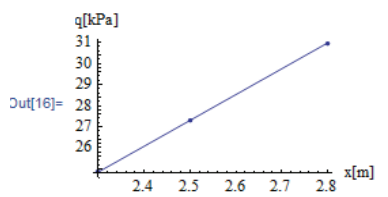

$\ln [17]:=\mathrm{d} 2835=\left\{\left\{2.8^{\circ}, 27.11^{\circ}\right\},\left\{3 .^{\prime}, 10.149999999999999^{\circ}\right\},\left\{3.5^{\prime},-32.3^{\prime}\right\}\right\} ;$

q2835 = LinearModelFit $[\mathrm{d} 2835,\{\mathrm{x}\}, \mathrm{x}]$;

q2835 ["BestFit"]

Jut[19] $=264.772-84.8769 \mathrm{x}$

$\ln [20]=\operatorname{Show}[\mathrm{Plot}[\mathrm{q} 2835[\mathrm{x}],\{\mathrm{x}, 2.8,3.5\}, \mathrm{PlotRange} \rightarrow \mathrm{All}], \operatorname{ListPlot}[\mathrm{d} 2835, \mathrm{PlotStyle} \rightarrow\{$ PointSize $[.02]\}]$, AxesLabel $\rightarrow\{$ "x $[\mathrm{m}] "$, , "q $[\mathrm{kPa}] "\}]$

$\mathrm{q}[\mathrm{kPa}]$




$\ln [21]:=d 354=\left\{\left\{3.5^{\circ}, 24.23\right\},\left\{4 .^{\circ}, 22.169999999999998^{\circ}\right\}\right\}$

q354 = LinearModelFit $[\mathrm{d} 354,\{\mathrm{x}\}, \mathrm{x}]$;

q354 ["BestFit"]

Out[23] $=38.65-4.12 x$

$\ln [24]:=\operatorname{Show}[P 1$ ot $[q 354[x],\{x, 3.5,4\}$, PlotRange $\rightarrow A l 1], \operatorname{ListPlot}[\mathrm{d} 354$, PlotStyle $\rightarrow\{$ PointSize $[.02]\}]$, AxesLabel $\rightarrow\{$ "x [m] ", "q[kPa]" $\}]$

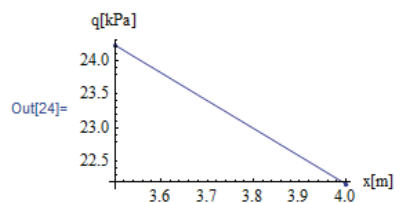

$\ln [2]]=d 441=\left\{\left\{4 .^{`}, 22.169999999999998^{`}\right\},\left\{41^{\prime}, 21.759999999999998^{\prime}\right\}\right\} ;$

$\mathrm{q} 441=$ LinearModelFit $[\mathrm{d} 441,\{\mathrm{x}\}, \mathrm{x}]$

q441 ["BestFit"]

Out $[27]=38.57-4.1 \times$

$\ln [28]=\operatorname{Show}[\mathrm{Plot}[\mathrm{q} 441[\mathrm{x}],\{\mathrm{x}, 4,4.1\}$, PlotRange $\rightarrow$ All $]$, ListPlot $[\mathrm{d} 441$, PlotStyle $\rightarrow\{$ PointSize $[.02]\}]$, AxesLabel $\rightarrow\{$ "x [m] ", "q[kPa]" $\}]$

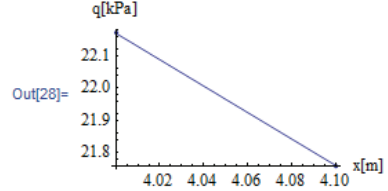

$\ln [29]:=d 4171=\left\{\left\{4.1^{\prime}, 29.259999999999998^{\prime}\right\},\left\{4.33^{\prime}, 29.36^{\prime}\right\},\left\{4.5^{`}, 29.449999999999996^{\prime}\right\},\left\{5{ }^{\prime}, 29.68^{\prime}\right\}\right.$,

$\left\{5.5^{`}, 29.909999999999997^{`}\right\},\left\{6^{`}, 30.15^{`}\right\},\left\{6.5^{`}, 30.380000000000003^{\prime}\right\},\left\{7 .^{`}, 30.6099999999999992^{`}\right\}$

$\left.\left\{7.1^{\circ}, 30.658^{\circ}\right\}\right\}$;

q4171 = LinearModelFit $[\mathrm{d} 4171,\{\mathrm{x}\}, \mathrm{x}]$

q4171 ["BestFit"]

Out[31] $=27.346+0.466597 x$

$\ln [32]=$ Show $[P 1 o t[q 4171[x],\{x, 4.1,7.1\}$, PlotRange $\rightarrow$ All $]$, ListPlot $[$ d4171, PlotStyle $\rightarrow\{$ PointSize $[.02]\}]$ AxesLabel $\rightarrow\{$ "x $[\mathrm{m}] "$, "q [kPa]" $\}]$

$\mathrm{q}[\mathrm{kPa}]$

Out[32]=

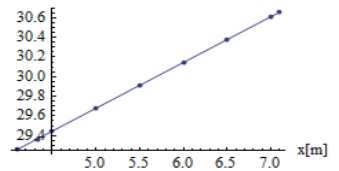

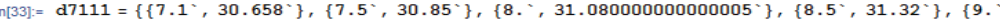

$\left.\left\{9.5^{\prime}, 31.779999999999994^{\prime}\right\},\left\{10 .^{\prime}, 32.02^{\prime}\right\},\left\{10.5^{`}, 32.25^{`}\right\},\left\{11 .^{\prime}, 32.480000000000004^{\prime}\right\}\right\} ;$

q7111 = LinearModelFit $[\mathrm{d} 7111,\{\mathrm{x}\}, \mathrm{x}]$;

q7111 ["BestFit"]

Out[35] $=27.3448+0.467139 x$

$\ln [36]:=$ Show $[P 1 o t[q 7111[x],\{x, 7.1,11\}$, PlotRange $\rightarrow$ All $]$, ListPlot $[d 7111$, PlotStyle $\rightarrow\{$ PointSize $[.02]\}]$ AxesLabel $\rightarrow\{$ "x $[\mathrm{m}] ", " \mathrm{q}[\mathrm{kPa}] "\}]$

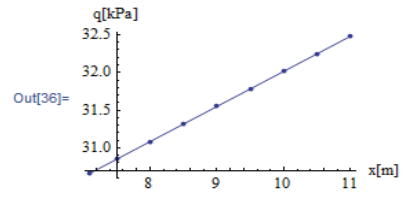

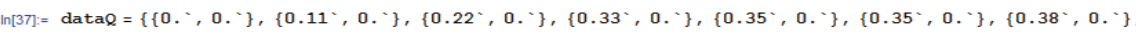

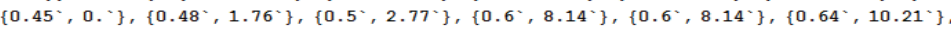



$\left\{1.52^{\circ}, 15.34^{\top}\right\},\left\{1.72^{\circ}, 17.81^{\top}\right\},\left\{1.72^{\prime}, 17.82^{\circ},\left\{2 ., 21.2^{\circ}\right\},\left\{2.3^{\prime}, 23.744^{\circ}\right\},\left\{2.5,27.3^{\circ}\right\}\right.$



$\left\{4.1^{`}, 21.759999999999998^{`}\right\},\left\{4.1^{\wedge}, 29.259999999999998^{`}\right\},\left\{4.33^{`}, 29.36^{`}\right\},\left\{4.5^{\star}, 29.449999999999996^{`}\right\}$

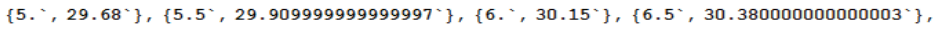

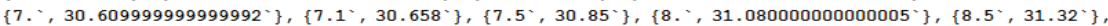

$\left\{9 .^{\prime}, 31.55000000000004^{\prime}\right\},\left\{9.5^{\prime}, 31.779999999999994^{\prime}\right\},\left\{10{ }^{\prime}, 32.02^{\prime}\right\},\left\{10.5^{\prime}, 32.25^{\prime}\right\}$,

$\left.\left\{11 .^{`}, 32.480000000000004^{`}\right\}\right\}$; 


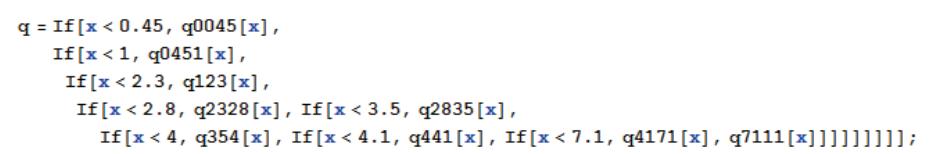

$\operatorname{In}[39]:=\mathrm{Pq}=\operatorname{Rotate}[$ Show $[\mathrm{Plot}[\{\mathrm{q}\},\{\mathrm{x}, 0,11\}, \mathrm{PlotStyle} \rightarrow\{$ Red $\}$, PlotRange $\rightarrow$ All $]$

ListPlot [dataQ, PlotStyle $\rightarrow$ \{PointSize[.01]\}], Frame $\rightarrow$ True, BaseStyle $\rightarrow\{$ FontWleight $\rightarrow$ "Normal", FontSize $\rightarrow 20\}$,

FrameLabel $\rightarrow\{\mathrm{x}[\mathrm{m}] ", " \mathrm{q}[\mathrm{kPa}]$ " $\}$, FrameTicks $\rightarrow$ All $],-90$ Degree $]$

$\mathrm{q}[\mathrm{kPa}]$

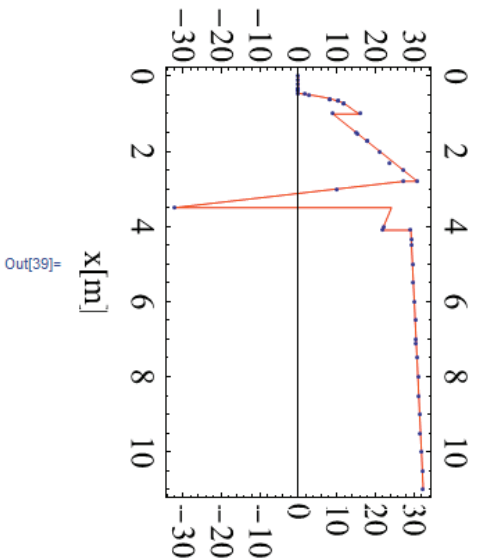

2. Solution of Euler - Bernouilli and Winkler equations.

$\ln [40]:=w 10=\operatorname{DSolve}\left[\mathrm{BJ} \mathrm{y}^{\prime}+\mathrm{H}^{\prime}[\mathrm{x}]=\mathrm{q0045}[\mathrm{x}], \mathrm{y}[\mathrm{x}], \mathrm{x}\right][[1]]$;

$\mathrm{w} 1=\mathrm{w} 10 / .\{\mathrm{C}[1] \rightarrow \mathrm{c} 1, \mathrm{C}[2] \rightarrow \mathrm{C} 2, \mathrm{C}[3] \rightarrow \mathrm{C} 3, \mathrm{C}[4] \rightarrow \mathrm{C} 4\} ;$

$\mathrm{w} 1=\mathrm{w} 1[[1]][[2]]$

Out[42] $=\mathrm{C} 1+\mathrm{C} 2 \mathrm{x}+\mathrm{C} 3 \mathrm{x}^{2}+\mathrm{C} 4 \mathrm{x}^{3}$

$\ln [43]=\mathrm{w} 20=\mathrm{DSolve}[\mathrm{BJ} \mathrm{y}+\mathrm{H}[\mathrm{x}]=\mathrm{q} 0451[\mathrm{x}], \mathrm{y}[\mathrm{x}], \mathrm{x}][[1]] ;$

$\mathrm{w} 2=\mathrm{w} 20 / .\{\mathrm{C}[1] \rightarrow \mathrm{C} 5, \mathrm{C}[2] \rightarrow \mathrm{C} 6, \mathrm{c}[3] \rightarrow \mathrm{C} 7, \mathrm{C}[4] \rightarrow \mathrm{C} 8\}$;

$\mathrm{w} 2=\mathrm{w} 2[[1]][[2]]$

Out[45] $=C 5+C 6 x+C 7 x^{2}+C 8 x^{3}+\frac{-3.48077 x^{4}+2.65004 x^{5}-0.998384 x^{6}+0.167845 x^{7}}{E J}$

$\ln [46]=\mathrm{w} 30=\operatorname{DSolve}\left[\mathrm{BJ} \mathrm{y}^{\prime}+\mathrm{H}[\mathrm{x}]=\mathrm{q} 123[\mathrm{x}], \mathrm{y}[\mathrm{x}], \mathrm{x}\right][[1]]$

$\mathrm{w} 3=\mathrm{w} 30 / .\{\mathrm{C}[1] \rightarrow \mathrm{C} 9, \mathrm{C}[2] \rightarrow \mathrm{C} 10, \mathrm{C}[3] \rightarrow \mathrm{C} 11, \mathrm{C}[4] \rightarrow \mathrm{C} 12\}$;

$\mathrm{w} 3=\mathrm{w} 3[[1]][[2]]$

Out[48] $=C 9+C 10 x+C 11 x^{2}+C 12 x^{3}+\frac{-0.135165 x^{4}+0.10189 x^{5}}{E J}$

$\ln [49]==w 40=D$ Solve $[\mathrm{EJ} y+\cdots[\mathrm{x}]=\mathrm{q} 2328[\mathrm{x}], \mathrm{y}[\mathrm{x}], \mathrm{x}][[1]]$;

$\mathrm{w} 4=\mathrm{w} 40 / .\{\mathrm{C}[1] \rightarrow \mathrm{c} 13, \mathrm{C}[2] \rightarrow \mathrm{C} 14, \mathrm{C}[3] \rightarrow \mathrm{C} 15, \mathrm{C}[4] \rightarrow \mathrm{C} 16\}$;

$w 4=w 4[[1]][[2]]$

Out[51] $=C 13+C 14 x+C 15 x^{2}+C 16 x^{3}+\frac{-0.135417 x^{4}+0.101842 x^{5}}{E J}$

$\ln [52]=\mathrm{w} 50=\mathrm{DSolve}[\mathrm{EJ} \mathrm{y}, \mathrm{W}[\mathrm{x}]=\mathrm{q} 2835[\mathrm{x}], \mathrm{y}[\mathrm{x}], \mathrm{x}][[1]]$;

$\mathrm{w} 5=\mathrm{w} 50 / .\{\mathrm{C}[1] \rightarrow \mathrm{C} 17, \mathrm{C}[2] \rightarrow \mathrm{C} 18, \mathrm{C}[3] \rightarrow \mathrm{C} 19, \mathrm{C}[4] \rightarrow \mathrm{C} 20\}$;

$\mathrm{w} 5=\mathrm{w} 5[[1]][[2]]$

Out[54] $=\mathrm{C} 17+\mathrm{C} 18 \mathrm{x}+\mathrm{C} 19 \mathrm{x}^{2}+\mathrm{C} 20 \mathrm{x}^{3}+\frac{11.0322 \mathrm{x}^{4}-0.707308 \mathrm{x}^{5}}{\text { EJ }}$

$\ln [55]==\mathrm{w} 60=\operatorname{DSolve}\left[\mathrm{EJ} \mathrm{y}^{\prime} \cdot \mathrm{H}[\mathrm{x}]+\mathrm{k} 2 * \mathrm{y}[\mathrm{x}]=\mathrm{q} 354[\mathrm{x}], \mathrm{y}[\mathrm{x}], \mathrm{x}\right][[1]]$;

$\mathrm{w} 6=\mathrm{w} 60 / .\{\mathrm{C}[1] \rightarrow \mathrm{C} 21, \mathrm{C}[2] \rightarrow \mathrm{C} 22, \mathrm{C}[3] \rightarrow \mathrm{C} 23, \mathrm{C}[4] \rightarrow \mathrm{C} 24\} ;$

$w 6=w 6[[1]][[2]]$

Out[57] $=-\frac{4.12(-9.38107+1 . \mathrm{x})}{\mathrm{k} 2}+\mathrm{C} 24 \mathrm{e}^{\frac{0.707107 \mathrm{k} 2^{1 / 4} \mathrm{x}}{\mathrm{EJ} \mathrm{J}^{1 / 4}}} \operatorname{Cos}\left[\frac{0.707107 \mathrm{k} 2^{1 / 4} \mathrm{x}}{\mathrm{EJ}^{1 / 4}}\right]+\mathrm{C} 21 \mathrm{e}^{-\frac{0.707107 \mathrm{k} 2^{1 / 4} \mathrm{x}}{\mathrm{EJ} \mathrm{J}^{1 / 4}}} \operatorname{Cos}\left[\frac{0.707107 \mathrm{k} 2^{1 / 4} \mathrm{x}}{\mathrm{EJ}^{1 / 4}}\right]+$

$\mathrm{C} 22 \mathrm{e}^{-\frac{0.707107 \mathrm{k} 2^{1 / 4} \mathrm{x}}{\mathrm{EJ} \mathrm{J}^{1 / 4}}} \operatorname{Sin}\left[\frac{0.707107 \mathrm{k} 2^{1 / 4} \mathrm{x}}{\mathrm{EJ}^{1 / 4}}\right]+\mathrm{C} 23 \mathrm{e}^{\frac{0.707107 \mathrm{k} 2^{1 / 4} \mathrm{x}}{\mathrm{EJ} J^{1 / 4}}} \operatorname{Sin}\left[\frac{0.707107 \mathrm{k} 2^{1 / 4} \mathrm{x}}{\mathrm{EJ}^{1 / 4}}\right]$ 
$\ln [58]:=\mathrm{w} 70=\mathrm{DSolve}\left[\mathrm{EJ} \mathrm{y}^{\prime}+\mathrm{H}^{\prime}[\mathrm{x}]+\mathrm{k} 2 * \mathrm{y}[\mathrm{x}]=\mathrm{q} 441[\mathrm{x}], \mathrm{y}[\mathrm{x}], \mathrm{x}\right][[1]] ;$

$\mathrm{w} 7=\mathrm{w} 70 / .\{\mathrm{C}[1] \rightarrow \mathrm{C} 25, \mathrm{C}[2] \rightarrow \mathrm{C} 26, \mathrm{C}[3] \rightarrow \mathrm{C} 27, \mathrm{C}[4] \rightarrow \mathrm{C} 28\} ;$

$w 7=w 7[1]][[2]]$

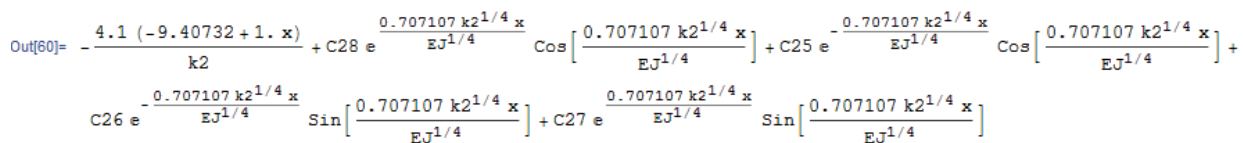

$\ln [61]:=w 80=\operatorname{DSolve}\left[\mathrm{EJ} \mathrm{y}^{\prime}+\cdots[\mathrm{x}]+\mathrm{k} 1 * \mathrm{y}[\mathrm{x}]=\mathrm{q} 4171[\mathrm{x}], \mathrm{y}[\mathrm{x}], \mathrm{x}\right][[1]]$;

$\mathrm{w} 8=\mathrm{w} 80 / .\{\mathrm{C}[1] \rightarrow \mathrm{C} 29, \mathrm{C}[2] \rightarrow \mathrm{C} 30, \mathrm{C}[3] \rightarrow \mathrm{C} 31, \mathrm{C}[4] \rightarrow \mathrm{c} 32\} ;$

$w 8=w 8[[1]][[2]]$

Out[63] $=\frac{0.466597(58.6073+1 . \mathrm{x})}{\mathrm{k} 1}+\mathrm{C} 32 \mathrm{e}^{\frac{0.707107 \mathrm{k} 1^{1 / 4} \mathrm{x}}{\mathrm{EJ} \mathrm{J}^{1 / 4}}} \operatorname{Cos}\left[\frac{0.707107 \mathrm{k} 1^{1 / 4} \mathrm{x}}{\mathrm{EJ}^{1 / 4}}\right]+\mathrm{C} 29 \mathrm{e}^{-\frac{0.707107 \mathrm{k} 1^{1 / 4} \mathrm{x}}{\mathrm{EJ} \mathrm{J}^{1 / 4}}} \operatorname{Cos}\left[\frac{0.707107 \mathrm{k} 1^{1 / 4} \mathrm{x}}{\mathrm{EJ} \mathrm{J}^{1 / 4}}\right]+$ $\mathrm{C} 30 \mathrm{e}^{-\frac{0.707107 \mathrm{k} 1^{1 / 4} \mathrm{x}}{\mathrm{EJ} \mathrm{J}^{1 / 4}}} \operatorname{Sin}\left[\frac{0.707107 \mathrm{k} 1^{1 / 4} \mathrm{x}}{\mathrm{EJ}^{1 / 4}}\right]+\mathrm{C} 31 \mathrm{e}^{\frac{0.707107 \mathrm{k} 1^{1 / 4} \mathrm{x}}{\mathrm{EJ} \mathrm{J}^{1 / 4}}} \operatorname{Sin}\left[\frac{0.707107 \mathrm{k} 1^{1 / 4} \mathrm{x}}{\mathrm{EJ}^{1 / 4}}\right]$

$\ln [64]=\mathrm{w} 90=\mathrm{DSolve}\left[\mathrm{EJ} \mathrm{y}^{\prime}+\mathrm{C}^{\prime}[\mathrm{x}]+\mathrm{k} 1 * \mathrm{y}[\mathrm{x}]=\mathrm{q} 7111[\mathrm{x}], \mathrm{y}[\mathrm{x}], \mathrm{x}\right][[1]] ;$

$\mathrm{w} 9=\mathrm{w} 90 / .\{\mathrm{c}[1] \rightarrow \mathrm{c} 33, \mathrm{C}[2] \rightarrow \mathrm{c} 34, \mathrm{c}[3] \rightarrow \mathrm{c} 35, \mathrm{C}[4] \rightarrow \mathrm{c} 36\} ;$

w9 $=w 9[[1]][[2]]$

Out[66] $=\frac{0.467139(58.5368+1 . \mathrm{x})}{\mathrm{k} 1}+\mathrm{C} 36 \mathrm{e}^{\frac{0.707107 \mathrm{k}^{1 / 4} \mathrm{x}}{\mathrm{EJ} \mathrm{J}^{1 / 4}}} \operatorname{Cos}\left[\frac{0.707107 \mathrm{k} 1^{1 / 4} \mathrm{x}}{\mathrm{EJ}^{1 / 4}}\right]+\mathrm{C} 33 \mathrm{e}^{-\frac{0.707107 \mathrm{k} 1^{1 / 4} \mathrm{x}}{\mathrm{EJ} J^{1 / 4}}} \operatorname{Cos}\left[\frac{0.707107 \mathrm{k} 1^{1 / 4} \mathrm{x}}{\mathrm{EJ} J^{1 / 4}}\right]+$ $\mathrm{C} 34 \mathrm{e}^{-\frac{0.707107 \mathrm{k} 1^{1 / 4} \mathrm{x}}{\mathrm{EJ} \mathrm{J}^{1 / 4}}} \operatorname{Sin}\left[\frac{0.707107 \mathrm{k} 1^{1 / 4} \mathrm{x}}{\mathrm{EJ}^{1 / 4}}\right]+\mathrm{C} 35 \mathrm{e}^{\frac{0.707107 \mathrm{k} 1^{1 / 4} \mathrm{x}}{\mathrm{EJ} J^{1 / 4}}} \operatorname{Sin}\left[\frac{0.707107 \mathrm{k} 1^{1 / 4} \mathrm{x}}{\mathrm{EJ}^{1 / 4}}\right]$

3. Loading beam material data and kh parameter

$\ln [67]:=\mathrm{EJ}=558000 ;$

4. Determination of the constants of integration.

$\ln [70]:=\operatorname{sol1}=$ Solve $[\{(D[w 1, x, x] / . x \rightarrow 0)=0,(D[w 1, x, x, x] / . x \rightarrow 0)=0,(D[w 9, x, x] / . x \rightarrow 11)=0$,

$(D[w 9, x, x, x] / . x \rightarrow 11)=0,(w 1 / . x \rightarrow 0.45)=(w 2 / . x \rightarrow 0.45),(D[w 1, x] / . x \rightarrow 0.45)=(D[w 2, x] / . x \rightarrow 0.45)$

$(\mathrm{D}[\mathrm{w} 1, \mathrm{x}, \mathrm{x}] / . \mathrm{x} \rightarrow 0.45)=(\mathrm{D}[\mathrm{w} 2, \mathrm{x}, \mathrm{x}] / . \mathrm{x} \rightarrow 0.45),(\mathrm{D}[\mathrm{w} 1, \mathrm{x}, \mathrm{x}, \mathrm{x}] / . \mathrm{x} \rightarrow 0.45)=(\mathrm{D}[\mathrm{w} 2, \mathrm{x}, \mathrm{x}, \mathrm{x}] / . \mathrm{x} \rightarrow 0.45)$,

$(\mathrm{w} 2 / \mathrm{x} \rightarrow 1)=(\mathrm{w} 3 / \mathrm{x} \rightarrow 1),(\mathrm{D}[\mathrm{w} 2, \mathrm{x}] / \mathrm{x} \rightarrow 1)=(\mathrm{D}[\mathrm{w} 3, \mathrm{x}] / \mathrm{x} \rightarrow 1),(\mathrm{D}[\mathrm{w} 2, \mathrm{x}, \mathrm{x}] / \mathrm{x} \rightarrow 1)=(\mathrm{D}[\mathrm{w} 3, \mathrm{x}, \mathrm{x}] / \mathrm{x} \rightarrow 1)$,

$(\mathrm{D}[\mathrm{w} 2, \mathrm{x}, \mathrm{x}, \mathrm{x}] / . \mathrm{x} \rightarrow 1)=(\mathrm{D}[\mathrm{w} 3, \mathrm{x}, \mathrm{x}, \mathrm{x}] / \mathrm{x} \rightarrow 1),(\mathrm{w} 3 / \mathrm{x} \rightarrow 2.3)=(\mathrm{w} 4 / \mathrm{x} \rightarrow 2.3)$,

$(D[w 3, x] / x \rightarrow 2.3)=(D[w 4, x] / x \rightarrow 2.3),(D[w 3, x, x] / x \rightarrow 2.3)=(D[w 4, x, x] / . x \rightarrow 2.3)$,

$(\mathrm{D}[\mathrm{w} 3, \mathrm{x}, \mathrm{x}, \mathrm{x}] / . \mathrm{x} \rightarrow 2.3)=(\mathrm{D}[\mathrm{w} 4, \mathrm{x}, \mathrm{x}, \mathrm{x}] / . \mathrm{x} \rightarrow 2.3),(\mathrm{w} 4 / . \mathrm{x} \rightarrow 2.8)=(\mathrm{w} 5 / . \mathrm{x} \rightarrow 2.8)$,

$(D[w 4, x] / x \rightarrow 2.8)=(D[w 5, x] / x \rightarrow 2.8),(D[w 4, x, x] / x \rightarrow 2.8)=(D[w 5, x, x] / x \rightarrow 2.8)$,

$(\mathrm{D}[\mathrm{w} 4, \mathrm{x}, \mathrm{x}, \mathrm{x}] / . \mathrm{x} \rightarrow 2.8)=(\mathrm{D}[\mathrm{w} 5, \mathrm{x}, \mathrm{x}, \mathrm{x}] / . \mathrm{x} \rightarrow 2.8),(\mathrm{w} 5 / . \mathrm{x} \rightarrow 3.5)=(\mathrm{w} 6 / . \mathrm{x} \rightarrow 3.5)$,

$(\mathrm{D}[\mathrm{w} 5, \mathrm{x}] / . \mathrm{x} \rightarrow 3.5)=(\mathrm{D}[\mathrm{w} 6, \mathrm{x}] / \mathrm{x} \rightarrow 3.5),(\mathrm{D}[\mathrm{w} 5, \mathrm{x}, \mathrm{x}] / . \mathrm{x} \rightarrow 3.5)=(\mathrm{D}[\mathrm{w} 6, \mathrm{x}, \mathrm{x}] / . \mathrm{x} \rightarrow 3.5)$,

$(D[w 5, x, x, x] / . x \rightarrow 3.5)=(D[w 6, x, x, x] / . x \rightarrow 3.5),(w 6 / . x \rightarrow 4)=(w 7 / . x \rightarrow 4)$,

$(\mathrm{D}[\mathrm{w} 6, \mathrm{x}] / . \mathrm{x} \rightarrow 4)=(\mathrm{D}[\mathrm{w} 7, \mathrm{x}] / . \mathrm{x} \rightarrow 4),(\mathrm{D}[\mathrm{w} 6, \mathrm{x}, \mathrm{x}] / . \mathrm{x} \rightarrow 4)=(\mathrm{D}[\mathrm{w} 7, \mathrm{x}, \mathrm{x}] / . \mathrm{x} \rightarrow 4)$,

$(\mathrm{D}[\mathrm{w} 6, \mathrm{x}, \mathrm{x}, \mathrm{x}] / \mathrm{x} \rightarrow 4)=(\mathrm{D}[\mathrm{w} 7, \mathrm{x}, \mathrm{x}, \mathrm{x}] / \mathrm{x} \rightarrow 4),(\mathrm{w} 7 / \mathrm{x} \rightarrow 4.1)=(\mathrm{w} 8 / \mathrm{x} \rightarrow 4.1)$,

$(\mathrm{D}[\mathrm{w} 7, \mathrm{x}] / . \mathrm{x} \rightarrow 4.1)=(\mathrm{D}[\mathrm{w} 8, \mathrm{x}] / . \mathrm{x} \rightarrow 4.1),(\mathrm{D}[\mathrm{w} 7, \mathrm{x}, \mathrm{x}] / \mathrm{x} \rightarrow 4.1)=(\mathrm{D}[\mathrm{w} 8, \mathrm{x}, \mathrm{x}] / . \mathrm{x} \rightarrow 4.1)$,

$(D[w 7, x, x, x] / x \rightarrow 4.1)=(D[w 8, x, x, x] / . x \rightarrow 4.1),(w 8 / . x \rightarrow 7.1)=(w 9 / . x \rightarrow 7.1), 1$

$(\mathrm{D}[\mathrm{w} 8, \mathrm{x}] / \mathrm{x} \rightarrow 7.1)=(\mathrm{D}[\mathrm{w} 9, \mathrm{x}] / \mathrm{x} \rightarrow 7.1),(\mathrm{D}[\mathrm{w} 8, \mathrm{x}, \mathrm{x}] / \mathrm{x} \rightarrow 7.1)=(\mathrm{D}[\mathrm{w} 9, \mathrm{x}, \mathrm{x}] / \mathrm{x} \rightarrow 7.1)$,

$(\mathrm{D}[\mathrm{w} 8, \mathrm{x}, \mathrm{x}, \mathrm{x}] / . \mathrm{x} \rightarrow 7.1)=(\mathrm{D}[\mathrm{w} 9, \mathrm{x}, \mathrm{x}, \mathrm{x}] / . \mathrm{x} \rightarrow 7.1)\}$

c1, c2 $, \mathrm{c} 3, \mathrm{c} 4, \mathrm{c} 5, \mathrm{c} 6, \mathrm{c} 7, \mathrm{c} 8, \mathrm{c} 9, \mathrm{c} 10, \mathrm{c} 11, \mathrm{c} 12, \mathrm{c} 13, \mathrm{c} 14, \mathrm{c} 15, \mathrm{c} 16, \mathrm{c} 17, \mathrm{c} 18, \mathrm{c} 19, \mathrm{c} 20, \mathrm{c} 21, \mathrm{c} 22$,

$\mathrm{c} 23, \mathrm{c} 24, \mathrm{c} 25, \mathrm{c} 26, \mathrm{c} 27, \mathrm{c} 28, \mathrm{c} 29, \mathrm{c} 30, \mathrm{c} 31, \mathrm{c} 32, \mathrm{c} 33, \mathrm{c} 34, \mathrm{c} 35, \mathrm{c} 36 \mathrm{\}}]$

Out[70] $=\left\{\left\{c 1 \rightarrow 0.0054068, c 2 \rightarrow-0.000809583, c 3 \rightarrow 3.79651 \times 10^{-20}, c 4 \rightarrow 0 ., c 5 \rightarrow 0.00540677, c 6 \rightarrow-0.000809217\right.\right.$,

$\mathrm{C} 7 \rightarrow-1.7591 \times 10^{-6}, \mathrm{C} 8 \rightarrow 4.44037 \times 10^{-6}, \mathrm{C} 9 \rightarrow 0.00540638, \mathrm{C} 10 \rightarrow-0.000808057, \mathrm{C} 11 \rightarrow-1.86846 \times 10^{-6}, \mathrm{C} 12 \rightarrow 8.66794 \times 10^{-7}$ $\mathrm{C} 13 \rightarrow 0.00540634, \mathrm{C} 14 \rightarrow-0.000807999, \mathrm{C} 15 \rightarrow-1.90384 \times 10^{-6}, \mathrm{C} 16 \rightarrow 8.75524 \times 10^{-7}, \mathrm{C} 17 \rightarrow 0.00563823, \mathrm{C} 18 \rightarrow-0.00122839$, $\mathrm{C} 19 \rightarrow 0.000302888, \mathrm{C} 20 \rightarrow-0.00010959, \mathrm{C} 21 \rightarrow 0.00320588, \mathrm{C} 22 \rightarrow 0.00360849, \mathrm{C} 23 \rightarrow 0.0000657, \mathrm{C} 24 \rightarrow-0.0000249667$, $\mathrm{C} 25 \rightarrow 0.00320877, \mathrm{C} 26 \rightarrow 0.00361008, \mathrm{C} 27 \rightarrow 0.0000654815, \mathrm{C} 28 \rightarrow-0.0000248459, \mathrm{C} 29 \rightarrow 0.00366111, \mathrm{c} 30 \rightarrow 0.00396743$, $\left.\left.\mathrm{C} 31 \rightarrow 3.6404 \times 10^{-6}, \mathrm{C} 32 \rightarrow 4.19128 \times 10^{-6}, \mathrm{C} 33 \rightarrow 0.00366147, \mathrm{C} 34 \rightarrow 0.0039673, \mathrm{C} 35 \rightarrow 3.63731 \times 10^{-6}, \mathrm{C} 36 \rightarrow 4.19641 \times 10^{-6}\right\}\right\}$ 


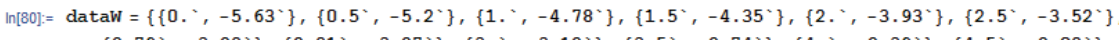
$\left\{2.79^{\circ},-3.28^{\circ}\right\},\left\{2.81^{\circ},-3.27^{\circ}\right\},\left\{3 .^{\circ},-3.12^{\circ}\right\},\left\{3.5^{\circ},-2.74^{\circ}\right\},\left\{4 .^{\circ},-2.39^{\circ}\right\},\left\{4.5^{\circ},-2.08^{\circ}\right\}$, $\left\{5 .{ }^{`},-1.82^{`}\right\},\left\{5.5^{`},-1.59^{`}\right\},\left\{6^{`},-1.41^{`}\right\},\left\{6.5^{`},-1.26^{`}\right\},\left\{7 .^{`},-1.15^{`}\right\},\left\{7.5^{\circ},-1.06^{`}\right\}$


ww $=$ If $[x<0.45$, ww 1 If $[x<1, w w 2$,

$\operatorname{If}[x<2.3, w w 3, \operatorname{If}[x<2.8, w w 4, \operatorname{If}[x<3.5, w w 5, \operatorname{If}[x<4, w w 6, \operatorname{If}[x<4.1, w w 7, \operatorname{If}[x<7.1, w w 8, w w 9]]]]]]]$;

$\ln [82]:=\operatorname{Pww}=\operatorname{Rotate}[$ Show $[\operatorname{Plot}[-w w * 1000,\{x, 0,11\}, \operatorname{PlotStyle} \rightarrow\{\operatorname{Red}\}, \operatorname{PlotRange} \rightarrow\{\{0,11\},\{0,-6\}\}]$, ListPlot [dataW, PlotStyle $\rightarrow$ PointSize[.015]\}], Frame $\rightarrow$ True, BaseStyle $\rightarrow$ FontWeight $\rightarrow$ "Normal", FontSize $\rightarrow 20\}$, FrameLabel $\rightarrow\{" x[m] ", " w[m m] "\}$, FrameTicks $\rightarrow$ All $],-90$ Degree $]$

$\mathrm{w}[\mathrm{mm}]$

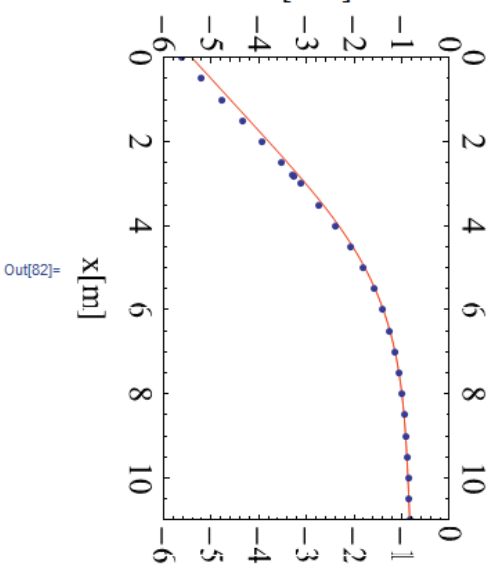

$\ln [83]:=w w 1 / . x \rightarrow 0$

Out[83] $=0.0054068$

$\ln [84]:=\operatorname{mom} 1=\mathrm{D}[\mathrm{ww} 1, \mathrm{x}, \mathrm{x}]$;

$\operatorname{mom} 2=\mathrm{D}[\mathrm{ww} 2, \mathrm{x}, \mathrm{x}]$

$\operatorname{mom} 3=\mathrm{D}[\mathrm{ww} 3, \mathrm{x}, \mathrm{x}]$;

$\operatorname{mom} 4=\mathrm{D}[\mathrm{ww} 4, \mathrm{x}, \mathrm{x}] ;$

$\operatorname{mom} 5=\mathrm{D}[\mathrm{ww} 5, \mathrm{x}, \mathrm{x}] ;$

$\operatorname{mom} 6=\mathrm{D}[\mathrm{ww} 6, \mathrm{x}, \mathrm{x}] ;$

$\operatorname{mom} 7=\mathrm{D}[\mathrm{ww} 7, \mathrm{x}, \mathrm{x}]$

$\operatorname{mom} 8=\mathrm{D}[\mathrm{ww} 8, \mathrm{x}, \mathrm{x}]$;

$\operatorname{mom} 9=\mathrm{D}[\mathrm{ww} 9, \mathrm{x}, \mathrm{x}] ;$

$\cos =$

If $[x<0.45, \operatorname{mom} 1$

If $[x<1, \operatorname{mom} 2$,

If $[x<2.3, \operatorname{mom} 3$

$\operatorname{If}[x<2.8, \operatorname{mom} 4, \operatorname{If}[x<3.5, \operatorname{mom} 5, \operatorname{If}[x<4, \operatorname{mom} 6, \operatorname{If}[x<4.1, \operatorname{mom} 7, \operatorname{If}[x<7.1, \operatorname{mom} 8, \operatorname{mom} 9]]]]]]] ;$

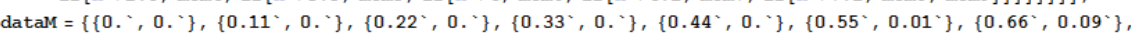



$\left\{1.43^{\circ}, 4.79^{\circ}\right\},\left\{1.54^{\circ}, 6.09^{\circ}\right\},\left\{1.65^{\circ}, 7.59^{\circ}\right\},\left\{1.76^{\circ}, 9.28^{\circ}\right\},\left\{1.87^{\circ}, 11.2^{\prime}\right\},\left\{1.98^{\circ}, 13.35^{\circ}\right\}$,

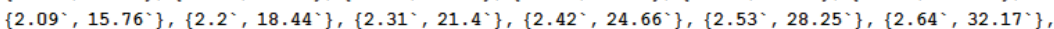



$\left\{3.19^{\circ}, 56.1^{\wedge}\right\},\left\{3.3^{\circ}, 61.12^{`}\right\},\left\{3.41^{\circ}, 65.95^{\circ}\right\},\left\{3.52^{\circ}, 70.48^{`}\right\},\left\{3.63^{\circ}, 74.52^{\circ}\right\},\left\{3.74^{\circ}, 78.19^{\prime}\right\}$

$\left\{3.85^{\circ}, 81.4^{\prime}\right\},\left\{3.96^{\circ}, 84.19^{\prime}\right\},\left\{4.07^{\circ}, 86.55^{\circ}\right\},\left\{4.18^{\circ}, 88.49^{\circ}\right\},\left\{4.29^{\circ}, 90.06^{\circ}\right\},\left\{4.4^{\circ}, 91.26^{\circ}\right\}$

$\left\{4.51^{`}, 92.11^{`}\right\},\left\{4.62^{`}, 92.65^{`}\right\},\left\{4.73^{\circ}, 92.88^{\prime}\right\},\left\{4.84^{\circ}, 92.83^{\prime}\right\},\left\{4.95^{\circ}, 92.52^{`}\right\},\left\{5.06^{\circ}, 91.96^{\prime}\right\}$,

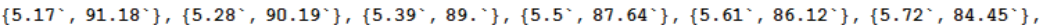

$\left\{5.83^{\circ}, 82.65^{\circ}\right\},\left\{5.94^{\circ}, 80.73^{\circ}\right\},\left\{6.05^{\circ}, 78.7^{`}\right\},\left\{6.16^{\circ}, 76.58^{\circ}\right\},\left\{6.27^{\circ}, 74.38^{`}\right\},\left\{6.38^{\circ}, 72.1^{\prime}\right\}$,

$\left\{6.49^{\circ}, 69.77^{\circ}\right\},\left\{6.6^{\circ}, 67.38^{\circ}\right\},\left\{6.71^{\circ}, 64.95^{\circ}\right\},\left\{6.82^{\circ}, 62.49^{\circ}\right\},\left\{6.93^{\circ}, 60.01^{\prime}\right\},\left\{7.04^{\circ}, 57.51^{\prime}\right\}$,

$\left\{7.15^{\circ}, 55 .^{\circ}\right\},\left\{7.26^{\circ}, 52.49^{\circ}\right\},\left\{7.37^{\circ}, 49.99^{\circ}\right\},\left\{7.48^{\circ}, 47.5^{\circ}\right\},\left\{7.59^{\circ}, 45.03^{\prime}\right\},\left\{7.7^{\circ}, 42.58^{\prime}\right\}$,

$\left\{7.81^{\prime}, 40.17^{`}\right\},\left\{7.92^{\circ}, 37.79^{\prime}\right\},\left\{8.03^{\circ}, 35.45^{\prime}\right\},\left\{8.14^{\circ}, 33.16^{\prime}\right\},\left\{8.25^{\circ}, 30.91^{\prime}\right\},\left\{8.36^{\circ}, 28.72^{\prime}\right\}$

$\left\{8.47^{\circ}, 26.59^{\circ}\right\},\left\{8.58^{\circ}, 24.51^{\circ}\right\},\left\{8.69^{\circ}, 22.5^{\circ}\right\},\left\{8.8^{\circ}, 20.56^{\circ}\right\},\left\{8.91^{\circ}, 18.69^{\circ}\right\},\left\{9.02^{\circ}, 16.89^{\circ}\right\}$,

$\left\{9.13^{\circ}, 15.17^{`}\right\},\left\{9.24^{\circ}, 13.53^{\circ}\right\},\left\{9.35^{\circ}, 11.97^{\circ}\right\},\left\{9.46^{\circ}, 10.5^{\circ}\right\},\left\{9.57^{\circ}, 9.11^{`}\right\},\left\{9.68^{\circ}, 7.81^{\wedge}\right\}$,

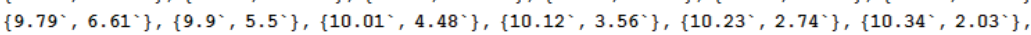

$\left.\left\{10.45^{`}, 1.42^{`}\right\},\left\{10.56^{\circ}, 0.91^{\prime}\right\},\left\{10.67^{\circ}, 0.52^{`}\right\},\left\{10.78^{`}, 0.23^{`}\right\},\left\{10.89^{`}, 0.06^{`}\right\},\left\{11 .^{`}, 0 .^{`}\right\}\right\}$; 
$\ln [95]=\operatorname{Pmom}=\operatorname{Rotate} \mid$ Show $\mid$ Plot $[\mathrm{BJ} * \operatorname{mom},\{\mathrm{x}, 0,11\}$, PlotStyle $\rightarrow\{$ Red $\}]$, ListPlot $[$ dataM, PlotStyle $\rightarrow\{$ PointSize $[.007]\}]$, PlotRange $\rightarrow$ All, Frame $\rightarrow$ True, BaseStyle $\rightarrow$ FontWeight $\rightarrow$ "Normal", FontSize $\rightarrow 20\}$, FrameLabel $\rightarrow\left\{\mathrm{x}[\mathrm{m}] ", \mathrm{M}\left[\frac{\mathrm{kNm}}{\mathrm{m}}\right] \mathrm{n}\right\}$, FrameTicks $\left.\rightarrow \mathrm{All}\right],-90$ Degree $]$

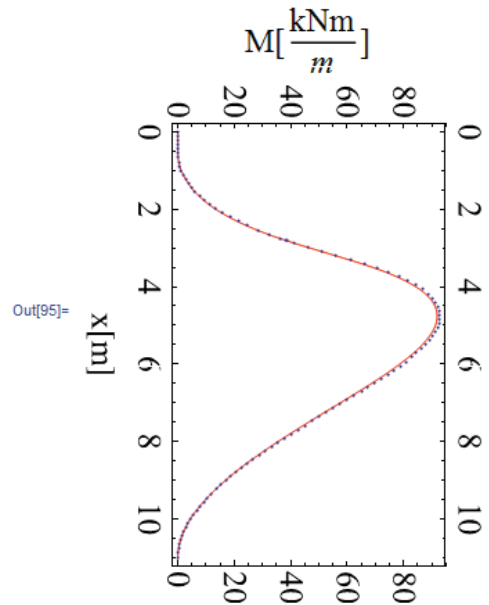

$\ln [96]:=$ FindMaximum $[\mathrm{EJ} * \operatorname{mom},\{\mathrm{x}, 4\}]$

Out[96] $=\{91.9052,\{x \rightarrow 4.7592\}\}$

$\ln [97]:=$ for $1=D[w w 1, x, x, x]$;

for $2=\mathrm{D}[\mathrm{ww} 2, \mathrm{x}, \mathrm{x}, \mathrm{x}]$;

for $3=\mathrm{D}[\mathrm{ww} 3, \mathrm{x}, \mathrm{x}, \mathrm{x}]$;

for $4=D[w w 4, x, x, x]$;

for $5=D[w w 5, x, x, x]$;

for6 $=\mathrm{D}[\mathrm{ww} 6, \mathrm{x}, \mathrm{x}, \mathrm{x}]$;

for $7=D[w w 7, x, x, x]$;

for8 $=\mathrm{D}[\mathrm{ww} 8, \mathrm{x}, \mathrm{x}, \mathrm{x}]$;

for $9=D[w w 9, x, x, x]$;

for $=$

If $[x<0.45$, for 1

If $[x<1$, for 2 ,

$\operatorname{If}[x<2.3$, for $3, \operatorname{If}[x<2.8$, for $4, \operatorname{If}[x<3.5$, for $5, \operatorname{If}[x<4$, for $6, \operatorname{If}[x<4.1$, for $7, \operatorname{If}[x<7.1$, for 8, for 9$]]]]]]]]$;

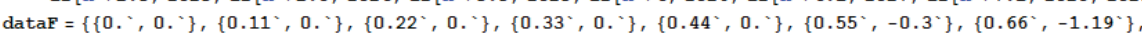

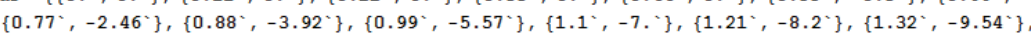

$\left\{1.43^{\circ},-11.03^{\circ}\right\},\left\{1.54^{\circ},-12.67^{\circ}\right\},\left\{1.65^{\circ},-14.46^{\circ}\right\},\left\{1.76^{\circ},-16.39^{\circ}\right\},\left\{1.87^{\circ},-18.48^{\circ}\right\}$,

$\left\{1.98^{\circ},-20.71^{\circ}\right\},\left\{2.09^{\circ},-23.09^{\circ}\right\},\left\{2.2^{\circ},-25.61^{\circ}\right\},\left\{2.31^{\circ},-28.29^{\circ}\right\},\left\{2.42^{\circ},-31.11^{\circ}\right\}$,

$\left\{2.53^{\circ},-34.08^{`}\right\},\left\{2.64^{\circ},-37.2^{\prime}\right\},\left\{2.75^{\circ},-40.46^{\circ}\right\},\left\{2.79^{\prime},-41.75^{\prime}\right\},\left\{2.81^{\prime},-42.21^{\prime}\right\}$,

$\left\{2.86^{\circ},-43.47^{`}\right\},\left\{2.97^{\circ},-45.38^{`}\right\},\left\{3.08^{\circ},-46.26^{\circ}\right\},\left\{3.19^{\circ},-46.11^{`}\right\},\left\{3.3^{\circ},-44.94^{`}\right\}$,

$\left\{3.41^{\circ},-42.74^{`}\right\},\left\{3.52^{\circ},-39.52^{\circ}\right\},\left\{3.63^{\circ},-35.47^{\circ}\right\},\left\{3.74^{\circ},-31.28^{\circ}\right\},\left\{3.85^{\circ},-27.24^{\circ}\right\}$,

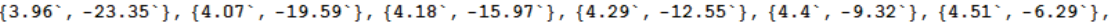

$\left\{4.62^{\circ},-3.46^{\circ}\right\},\left\{4.73^{\circ},-0.8^{\circ}\right\},\left\{4.84^{\circ}, 1.67^{\circ}\right\},\left\{4.95^{\circ}, 3.98^{`}\right\},\left\{5.06^{\circ}, 6.11^{`}\right\},\left\{5.17^{\circ}, 8.09^{\circ}\right\}$,

$\left\{5.28^{\circ}, 9.92^{\circ}\right\},\left\{5.39^{\circ}, 11.59^{`}\right\},\left\{5.5^{\circ}, 13.13^{\circ}\right\},\left\{5.61^{\circ}, 14.53^{\circ}\right\},\left\{5.72^{\circ}, 15.8^{\circ}\right\},\left\{5.83^{\circ}, 16.94^{`}\right\}$,

$\left\{5.94^{\circ}, 17.96^{\circ}\right\},\left\{6.05^{\circ}, 18.88^{\circ}\right\},\left\{6.16^{\circ}, 19.68^{\circ}\right\},\left\{6.27^{\circ}, 20.38^{\circ}\right\},\left\{6.38^{\circ}, 20.98^{\circ}\right\},\left\{6.49^{\circ}, 21.48^{\circ}\right\}$,

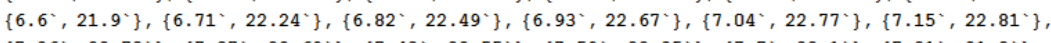

$\left\{7.26^{\circ}, 22.78^{`}\right\},\left\{7.37^{\circ}, 22.69^{\circ}\right\},\left\{7.48^{\circ}, 22.55^{\circ}\right\},\left\{7.59^{\circ}, 22.35^{`}\right\},\left\{7.7^{`}, 22.1^{`}\right\},\left\{7.81^{\circ}, 21.8^{`}\right\}$,

$\left\{7.92^{\circ}, 21.46^{\circ}\right\},\left\{8.03^{\circ}, 21.07^{\circ}\right\},\left\{8.14^{\circ}, 20.64^{\circ}\right\},\left\{8.25^{\circ}, 20.17^{\circ}\right\},\left\{8.36^{\circ}, 19.67^{\circ}\right\},\left\{8.47^{\circ}, 19.13^{\circ}\right\}$,

$\left\{8.58^{\circ}, 18.56^{\circ}\right\},\left\{8.69^{\circ}, 17.96^{\circ}\right\},\left\{8.8^{\circ}, 17.33^{\circ}\right\},\left\{8.91^{\circ}, 16.67^{\circ}\right\},\left\{9.02^{\circ}, 15.99^{\circ}\right\},\left\{9.13^{\circ}, 15.28^{\prime}\right\}$,

$\left\{9.24^{\circ}, 14.55^{\circ}\right\},\left\{9.35^{\circ}, 13.79^{\circ}\right\},\left\{9.46^{\circ}, 13.01^{\circ}\right\},\left\{9.57^{\circ}, 12.21^{\circ}\right\},\left\{9.68^{\circ}, 11.39^{\circ}\right\},\left\{9.79^{\circ}, 10.54^{\circ}\right\}$,

$\left\{9.9^{\circ}, 9.68^{\circ}\right\},\left\{10.01^{\circ}, 8.8^{\circ}\right\},\left\{10.12^{\circ}, 7.89^{\circ}\right\},\left\{10.23^{\circ}, 6.97^{\circ}\right\},\left\{10.34^{\circ}, 6.03^{\circ}\right\},\left\{10.45^{\circ}, 5.07^{\circ}\right\}$,

$\left.\left\{10.56^{`}, 4.1^{`}\right\},\left\{10.67^{\circ}, 3.1^{`}\right\},\left\{10.78^{`}, 2.08^{`}\right\},\left\{10.89^{`}, 1.05^{`}\right\},\left\{11 .^{`}, 0 .^{`}\right\}\right\}$;

$\ln [108]:=\operatorname{Pfor}=\operatorname{Rotate}[\operatorname{Show}[\operatorname{Plot}[-\mathrm{EJ} *$ for $,\{\mathrm{x}, 0,11\}, \operatorname{PlotStyle} \rightarrow\{\operatorname{Red}\}], \operatorname{ListPlot}[\operatorname{dataF}, \operatorname{PlotStyle} \rightarrow\{$ PointSize $[.007]\}]$,

PlotRange $\rightarrow$ All, Frame $\rightarrow$ True, BaseStyle $\rightarrow\{$ FontWeight $\rightarrow$ "Normal", FontSize $\rightarrow 20\}$,

FrameLabel $\rightarrow\left\{n \mathrm{x}[\mathrm{m}] ", " \mathrm{~T}\left[\frac{\mathrm{kN}}{\mathrm{m}}\right] n\right\}$, FrameTicks $\left.\rightarrow \mathrm{All}\right],-90$ Degree $]$ 




\section{REFERENCES}

[1] BAJdA M., KodA E., Badania geotechniczne do oceny warunków w strefach przykrawędziowych Skarpy Warszawskiej. Przegląd Naukowy Inżynieria i Kształtowanie Środowiska, 2013, 60, 126-136, (in Polish).

[2] Braja M., DAS, Principles of Geotechnical Engineering, Cengage Learning Thomson ed., Toronto, 2010.

[3] Chadeisson R., Parois continues moulees dans le sols, Proceedings of the 5th European Conference on Soil Mechanics and Foundation Engineering, Dunod, Paris, 1961, Vol. 2, 563-568.

[4] FINE Ltd. 2013. GEO5 - Podręcznik użytkownika. Wersja 17.

[5] LIPIŃSKI M.J., WDOWSKA M.K., A stress history and strain dependent stiffness of overconsolidated cohesive soil, Annals of Warsaw University of Life Sciences - SGGW, Land Reclamation, 2011, 43(2), 207-216.

[6] Ou Chang-Yu, Deep Excavation Theory and Practice, Taylor \& Francis Group, London, 2006.

[7] PASIK T., Koda E., Analiza sit wewnętrznych i przemieszczeń rozpieranej ściany szczelinowej, Acta Scientiarum Polonorum - Architectura, 2013, 12(4), 121-133, (in Polish).
[8] PN-83/B-03010 Ściany oporowe. Obliczenia statyczne i projektowanie, (in Polish).

[9] PN-EN 1997-1 Eurokod 7. Projektowanie geotechniczne. Część 1: Zasady ogólne, (in Polish).

[10] RedA A., Contribution à l'étude des problèmes du creusement avec bouclier à pression de terre, Thèse de Doctorat. Institut National des Sciences Appliquées, Lyon, 1994.

[11] SiemińsKa-Lewandowska A., Mitew-Czajewska M., Design of deep excavations according to Eurocode 7, Studia Geotechnica et Mechanica, 2008, 30 (1-2), 207-214.

[12] SiemińSKA-LewANDOwsKa A., Glębokie wykopy. Projektowanie $i$ wykonawstwo, WKŁ, Warszawa, 2011, (in Polish).

[13] Wellin P., Programming with Mathematica. An Introduction, Cambridge University Press, 2013.

[14] WrZESIŃSKI G., LECHOWICZ Z., Influence of the rotation of principal stress directions on undrained shear strength, Annals of Warsaw University of Life Sciences - SGGW. Land Reclamation, 2013, 45(2), 183-192.

[15] Wysokiński L., Kotlicki W., Godlewski T., Projektowanie geotechniczne wedlug Eurokodu 7, ITB, Warszawa, 2011, (in Polish). 